\title{
Transcatheter Aortic Valve Implantation (TAVI) in Singapore: Cost Effective After a Decade of Evolution?
}

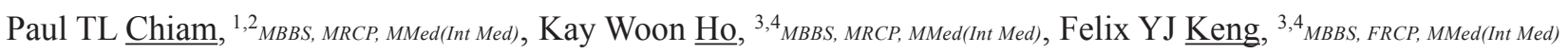

Aortic stenosis (AS) is a common valvular disease in developed countries and when untreated, symptomatic severe AS can result in a $50 \%$ mortality at $1-2$ years. ${ }^{1}$ Transcatheter aortic valve implantation or replacement (TAVI or TAVR) was first performed as an alternative to surgical aortic valve replacement (SAVR) in a no-option patient. ${ }^{2}$ Clinical trials, starting with inoperable severe AS patients, showed that TAVI reduced mortality when compared to medical therapy, ${ }^{1}$ and was non-inferior or even superior to SAVR in high or intermediate risk patients. ${ }^{3-6}$ More recently, TAVI has also been shown to be superior-with a lower major stroke or death and rehospitalisation rate-to SAVR in low risk patients. ${ }^{7,8}$ Since TAVI was first performed in Singapore (and Asia) in 2009, ${ }^{9}$ the technology has evolved, and third generation devices are currently in use with improved clinical outcomes. ${ }^{10}$

With these expanding indications, TAVI will be increasingly performed in an enlarging pool of patients with severe AS. The attractiveness of TAVI lies in the percutaneous nature of the procedure (virtually all cases are now performed via a transfemoral access) and the short recovery period and hospital stay (2-3 days on average vs 7-8 days for SAVR). However, the major limitation to the greater adoption of this therapy is the cost of the transcatheter bioprosthesis. ${ }^{10}$ Currently at SGD35,000-40,000, it is significantly more costly than a surgical bioprosthesis (SGD4,000-5,000). Thus, the cost effectiveness of TAVI vis-a-vis SAVR becomes a paramount consideration. Although the individual patient will undoubtedly prefer a less invasive approach, healthcare economics is likely to influence the uptake of this technology.

Previous studies examining the cost effectiveness of TAVI showed that it was cost effective as compared to medical therapy in inoperable patients. ${ }^{11,12}$ However, the cost effectiveness in high and intermediate risk patients were less certain, with the data from randomised trials favouring TAVI ${ }^{13-15}$ whereas real world data were mixed. ${ }^{16-21}$ However, these data were based on both femoral (true percutaneous) and alternative access routes (eg. transapical or direct aortic, both of which require a mini thoracotomy), and the transcatheter prostheses were of older iterations. More recent data in low-intermediate risk patients or using the latest (third) generation prostheses have demonstrated that TAVI is more cost effective than SAVR. ${ }^{22-23}$

In this issue of the journal, Kuntjoro et al. (page 423) describes the cost effectiveness of TAVI vs SAVR in a Singapore healthcare setting. Their report is one of the few describing an important health economics issue surrounding TAVI in an Asian healthcare system, data of which are lacking outside Japan. ${ }^{19,20}$ A local study is essential to evaluate factors unique to the Singapore healthcare system which may affect the cost-benefit ratio of TAVI vs SAVR. Although most clinicians are unfamiliar with such cost analyses, it is increasingly important to understand these issues with advancement in (expensive) medical technology and devices.

The authors used outcome data from the PARTNER 2A trial (a randomised trial of TAVI vs SAVR using the balloon-expandable Edwards Sapien XT valve) and the PARTNER 3 cohort trial (a post marketing observational registry using the third generation balloon-expandable Edwards Sapien 3 valve), to construct a Markov and decision tree model using costs derived from a local healthcare institution.

Using QALY (quality adjusted life years-1 QALY equates to 1 year in perfect health) and ICER (incremental cost effectiveness ratio-defined by increase in costs of TAVI over SAVR for 1 QALY), the authors showed that TAVI generated an additional 0.19 QALY, and was more cost effective than SAVR in $98 \%$ of scenarios, with an ICER of $\approx$ SGD33,000 (presumes a "willingness-topay" per QALY gained based on Singapore's per capita GDP of $\approx$ SGD73,000). TAVI was found to be highly

\footnotetext{
${ }^{1}$ The Heart and Vascular Centre, Mount Elizabeth Medical Centre, Singapore.

${ }^{2}$ Yong Loo Lin School of Medicine, National University of Singapore.

${ }^{3}$ Department of Cardiology, National Heart Centre Singapore, Singapore.

${ }^{4}$ Duke-NUS Medical School, Singapore, Singapore.

Address for Correspondence: Adj. A/Prof Paul TLChiam, The Heart and Vascular Centre, Mount Elizabeth Hospital, 3 MountElizabeth, \#08-06, Singapore 228510 Email: paulchiam@heartvascularcentre.com
} 
cost-effective assuming patients lived an average of 8 extra years. Even if that was shortened to 5 years, TAVI was still found to be cost effective, with an ICER of $\approx$ SGD60,000. The cost effectiveness of TAVI became more pronounced if data from the PARTNER 3 registry (with better clinical outcomes) were used with an ICER of $\approx$ SGD21,000 and SGD44,000 at 8 and 5 years respectively. However, the actual amount that would be considered incrementally cost effective may be quite different at a national healthcare level. To put things in perspective, the corresponding ICER for TAVI has been suggested to be USD50,000-100,000 and $£ 20,000-30,000$ for the United States and the United Kingdom respectively.

Previous cost effectiveness studies were based on the North American or European healthcare systems which are markedly different from a Singapore (Asian) setting. For instance, manpower costs (e.g. nursing and ancillary staff) are known to be much greater in the Western hemisphere than in most parts of Asia. Thus, important considerations such as length of hospital stay may take on more prominence in the West when comparing cost effectiveness.

The authors based their outcome data on the PARTNER 2A trial, which is a highly controlled environment with carefully selected patients. Although this is an accepted method of assessing cost effectiveness used by many researchers in the West, using these data in a Singapore healthcare setting may not reflect the actual outcomes and costs in our population. Perhaps it could have been more informative if the authors employed propensity matching of local patients to compare costs of TAVI vs SAVR, as utilised in some reports. ${ }^{17,21}$ Another major drawback of the study is the fact that the authors used the 2-year outcome data of the PARTNER 2A trial that showed superiority of transfemoral TAVI, with a lower death or disabling stroke rate, as compared to SAVR. ${ }^{5}$ The recently published 5-year outcome of the PARTNER 2A trial showed however, that there was no longer any significant difference in the incidence of death of disabling stroke between TAVI and SAVR. ${ }^{26}$ In addition, repeat hospitalisations and aortic valve reinterventions were more frequent with TAVI ${ }^{26}$ Hence, the ICER of TAVI in this study may have been inflated in favour of TAVI, thereby weakening the study conclusions. There were also other major assumptions. Residual paravalvular aortic regurgitation and permanent pacemaker implantation, both of which occur more frequently with TAVI and may increase long-term costs, were not included in the model and may have affected the results. Nevertheless, this current report sheds light on the local cost effectiveness of a rapidly growing and evolving technology.

Several factors are likely to contribute to further reducing the long-term cost and enhancing the cost effectiveness of TAVI. First, as the proportion of low or intermediate risk patients increase (these patients generally have a longer life expectancy), TAVI is likely to become more cost effective than SAVR (assuming the durability of the transcatheter and surgical bioprosthesis is similar). Secondly, design improvements of transcatheter prostheses (both balloon-expandable and self-expanding) and use of the femoral approach have vastly improved TAVI outcomes (e.g. low stroke or death rate, less residual paravalvular aortic regurgitation and permanent pacemaker implantation). Thirdly, and most importantly, the cost of the transcatheter prosthesis will gradually decrease (due to competition from upcoming technologies, economies of scale as the procedure becomes increasingly performed, and because current transcatheter prostheses are priced at a premium in Asia as compared to European benchmarks), ${ }^{10}$ driving the cost effectiveness in favour of TAVI.

This technology has proven to be an attractive therapeutic option in certain groups of patients with severe AS in the short to medium term, although long-term (10-15 years) data, including valve durability, are currently sparse. This paper may serve as a guide for further analyses on the most cost-effective funding strategies for TAVI. It is important to bear in mind however, that such studies are population based which do not consider individual factors in their models. Thus, the heart valve team has a central role to assess all factors, including cost-effectiveness, to choose the most appropriate therapy and achieve the best possible outcome for the individual patient.

\section{REFERENCES}

1. Leon MB, Smith CR, Mack M et al. Transcatheter aortic-valve implantation for aortic stenosis in patients who cannot undergo surgery. N Engl J Med 2010;363:1597-607.

2. Cribier A, Eltchaninoff H, Bash A et al. Percutaneous transcatheter implantation of an aortic valve prosthesis for calcific aortic stenosis: first human case description. Circulation 2002;106:3006-8.

3. Smith $\mathrm{CR}$, Leon MB, Mack MJ et al. Transcatheter versus surgical aortic-valve replacement in high-risk patients. N Engl J Med 2011;364:2187-98.

4. Adams DH, Popma JJ, Reardon MJ et al. Transcatheter aorticvalve replacement with a self-expanding prosthesis. N Engl J Med 2014;370:1790-8. 
5. Leon MB, Smith CR, Mack MJ et al. Transcatheter or Surgical Aortic-Valve Replacement in Intermediate-Risk Patients. N Engl J Med 2016;374:1609-20.

6. Reardon MJ, Van Mieghem NM, Popma JJ et al. Surgical or Transcatheter Aortic-Valve Replacement in Intermediate-Risk Patients. N Engl J Med 2017;376:1321-1331.

7. Mack MJ, Leon MB, Thourani VH et al. Transcatheter Aortic-Valve Replacement with a Balloon-Expandable Valve in Low-Risk Patients. N Engl J Med 2019;380:1695-1705.

8. Popma JJ, Reardon MJ. Transcatheter Aortic-Valve Replacement in Low-Risk Patients. Reply. N Engl J Med 2019;381:685.

9. Chiam PT, Koh TH, Chao VT et al. Percutaneous transcatheter aortic valve replacement: first transfemoral implant in Asia. Singapore Med J 2009;50:534-7.

10. Chiam PTL. Transcatheter aortic valve implantation in Asia: the first decade. EuroIntervention 2018;14:35-37.

11. Reynolds MR, Magnuson EA, Wang K et al. Cost-effectiveness of transcatheter aortic valve replacement compared with standard care among inoperable patients with severe aortic stenosis: results from the placement of aortic transcatheter valves (PARTNER) trial (Cohort B). Circulation 2012;125:1102-9.

12. Hancock-Howard RL, Feindel CM, Rodes-Cabau J, Webb JG, Thompson AK, Banz K. Cost effectiveness of transcatheter aortic valve replacement compared to medical management in inoperable patients with severe aortic stenosis: Canadian analysis based on the PARTNER Trial Cohort B findings. J Med Econ 2013;16:566-74.

13. Reynolds MR, Magnuson EA, Lei Y et al. Cost-effectiveness of transcatheter aortic valve replacement compared with surgical aortic valve replacement in high-risk patients with severe aortic stenosis: results of the PARTNER (Placement of Aortic Transcatheter Valves) trial (Cohort A). J Am Coll Cardiol 2012;60:2683-92.

14. Reynolds MR, Lei Y, Wang K et al. Cost-Effectiveness of Transcatheter Aortic Valve Replacement With a Self-Expanding Prosthesis Versus Surgical Aortic Valve Replacement. J Am Coll Cardiol 2016;67:29-38

15. Baron SJ, Wang K, House JA et al. Cost-Effectiveness of Transcatheter Versus Surgical Aortic Valve Replacement in Patients With Severe Aortic Stenosis at Intermediate Risk. Circulation 2019; 139:877-888.
16. Kularatna S, Byrnes J, Mervin MC, Scuffham PA. Health Technology Assessments Reporting Cost-Effectiveness of Transcatheter Aortic Valve Implantation. Int J Technol Assess Health Care 2016;32:89-96.

17. Ailawadi G, LaPar DJ, Speir AM et al. Contemporary Costs Associated With Transcatheter Aortic Valve Replacement: A Propensity-Matched CostAnalysis. Ann Thorac Surg 2016;101:154-60; discussion 160 .

18. Tam DY, Hughes A, Wijeysundera HC, Fremes SE. Cost-Effectiveness of Self-Expandable Transcatheter Aortic Valves in Intermediate-Risk Patients. Ann Thorac Surg 2018;106:676-683.

19. Inoue $\mathrm{S}$, Nakao $\mathrm{K}$, Hanyu $\mathrm{M}$ et al. Cost-Effectiveness of Transcatheter Aortic Valve Implantation Using a BalloonExpandable Valve in Japan: Experience From the Japanese Pilot Health Technology Assessment. Value Health Reg Issues 2020;21:82-90.

20. Kodera S, Kiyosue A, Ando J, Komuro I. Cost effectiveness of transcatheter aortic valve implantation in patients with aortic stenosis in Japan. J Cardiol 2018;71:223-229.

21. Osnabrugge RL, Head SJ, Genders TS et al. Costs of transcatheter versus surgical aortic valve replacement in intermediate-risk patients. Ann Thorac Surg 2012;94:1954-60.

22. Goodall G, Lamotte M, Ramos M, Maunoury F, Pejchalova B, de Pouvourville G. Cost-effectiveness analysis of the SAPIEN 3 TAVI valve compared with surgery in intermediate-risk patients. J Med Econ 2019;22:289-296.

23. Zhou J, Liew D, Duffy SJ, Walton A, Htun N, Stub D. Cost-effectiveness of transcatheter aortic valve implantation compared to surgical aortic valve replacement in the intermediate surgical risk population. Int J Cardiol 2019;294:17-22.

24. Geisler BP, Jorgensen TH, Thyregod HGH, Pietzsch JB, Sondergaard L. Cost-effectiveness of transcatheter versus surgical aortic valve replacement in patients at lower surgical risk: results from the NOTION trial. EuroIntervention 2019;15:e959-e967.

25. Tarride JE, Luong T, Goodall G, Burke N, Blackhouse G. A Canadian cost-effectiveness analysis of SAPIEN 3 transcatheter aortic valve implantation compared with surgery, in intermediate and high-risk severe aortic stenosis patients. Clinicoecon Outcomes Res 2019;11:477-486. 